\title{
Driver Readiness Model for Regulating the Transfer from Automation to Human Control
}

\author{
Tina Mioch \\ TNO \\ Kampweg 5 \\ 3769 DE Soesterberg \\ The Netherlands \\ Tina.Mioch@tno.nl
}

\author{
Liselotte Kroon \\ TNO \\ Kampweg 5 \\ 3769 DE Soesterberg \\ The Netherlands \\ Liselotte.Kroon@tno.nl
}

\author{
Mark A. Neerincx \\ TNO \\ Kampweg 5 \\ 3769 DE Soesterberg \\ The Netherlands \\ Mark.Neerincx@tno.nl
}

\begin{abstract}
In the collaborative driving scenario of truck platooning, the first car is driven by its chauffeur and the next cars follow automatically via a so-called 'virtual tow-bar'. The chauffeurs of the following cars do not drive 'in the towbar mode', but need to be able to take back control in foreseen and unforeseen conditions. It is crucial that this transfer of control only takes place when the chauffeur is ready for it. This paper presents a Driver Readiness (DR) ontological model that specifies the core factors, with their relationships, of a chauffeur's current and near-future readiness for taking back the control of driving. A first model was derived from a literature study and an analysis of truck driving data, which was refined subsequently based on an expert review. This DR model distinguishes (a) current and required states for the physical (hand, feet, head, and seating position) and mental readiness (attention and situation awareness), (b) agents (human and machine actor), (c) policies for agent behaviors, and (d) states of the vehicle and its environment. It provides the knowledge base of a Control Transfer Support (CTS) agent that assesses the current and predicted chauffeur state and guides the transition of control in an adaptive and personalized manner. The DR model will be fed by information from the network and in-car sensors. The behaviors of the CTS agent will be generated and constrained by the instantiated policies, providing an important step towards a safe transfer of control from automation to human driver.
\end{abstract}

\section{Author Keywords}

Ontology; Collaborative driving; Driver readiness; Transfer of control; Reusability; Knowledge representation.

\section{ACM Classification Keywords}

H.1.2. Models and Principles: User/Machine Systems - Human factors; I.2.4 Artificial Intelligence: Knowledge Representation Formalisms and Methods - Representations (procedural and rule-based), Semantic networks

Permission to make digital or hard copies of all or part of this work for personal or classroom use is granted without fee provided that copies are not made or distributed for profit or commercial advantage and that copies bear this notice and the full citation on the first page. Copyrights for components of this work owned by others than ACM must be honored. Abstracting with credit is permitted. To copy otherwise, or republish, to post on servers or to redistribute to lists, requires prior specific permission and/or a fee. Request permissions from permissions@ acm.org.

IUI 2017, March 13-16, 2017, Limassol, Cyprus.

Copyright (c) 2017 ACM 978-1-4503-4348-0/17/03\$15.00.

http://dx.doi.org/10.1145/3025171.3025199

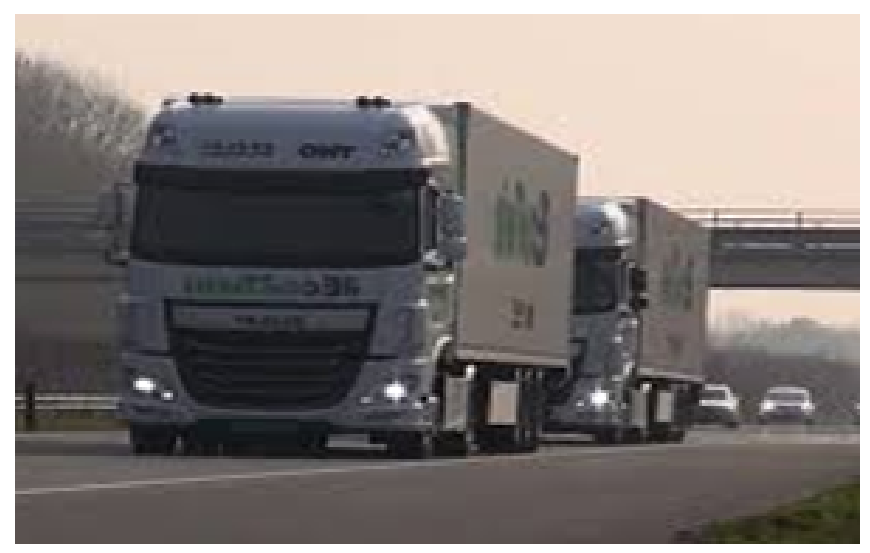

Figure 1. Truck platooning experiment with a CTS agent.

\section{INTRODUCTION}

Collaborative driving, such as truck platooning, is one of the likely scenarios for implementing autonomous driving in the near future. The required technology is mature and the benefits are substantial (energy-saving, chauffeur off-loading). In this scenario, trucks closely follow each other via a 'virtual tow-bar' and communicate plan, state \& observational data. The first truck in the platoon is controlled by a chauffeur, while the next trucks follow the first truck (actually, they know the 'intentions' of the first truck and can, consequently, anticipate the resulting truck behavior). After giving control to the truck platoon system, the chauffeurs in the following trucks are not driving anymore (see Figure 1).

When approaching the destination or the planned point for separating the way, and when requested by the first chauffeur or platoon system for other reasons, the chauffeur needs to take back control. It is crucial that he or she is in the right state, with enough time and relevant information, for this transfer of control [12].

This paper presents a Driver Readiness (DR) model that specifies the core factors, with their relationships, of chauffeur's current and near-future readiness for taking back the control of driving. To establish a knowledge base that can be shared and used by human and machine, we specify the DR model in an ontology: An explicit, structured, and semantically rich representation of declarative knowledge. Similar to cognitive theories, the DR ontology will distinguish upper level ('frames' or 'classes') from lower level knowledge ('instan- 
tiations') to represent context-dependent behavioral patterns for transfer of control (cf. [16, 14]).The DR ontology will enable communication between the driver and the automation about driver's readiness state.

Furthermore, the DR model provides the knowledge-base of a sensing and reasoning agent that assesses a driver's momentary state and supports the transfer from automation to manual control. This Control Transfer Support (CTS) agent assesses the current and predicted chauffeur state and guides the transition of control according to the safety and comfort norms. We develop specific policies for the CTS agent to enforce the required adaptive norm-compliant behaviors (e.g., providing an early warning to give sufficient time, and to remind the driver to look in the rear mirror to gain a better situation awareness). The concepts of these policies (like 'warning' and 'situation awareness') are defined in the ontology, as a basis for the agent's reasoning. This way, the agent's knowledge base and reasoning is transparent and can be explained to the chauffeurs to evoke the right behavioral responses and trust level.

The main contribution of this paper is the identification, structuring, and formalization of the readiness concepts and interrelationships into a coherent model (i.e., a univocal ontology and concise set of policies). The ontology and policies are derived theory and practice, developed in an iterative and incremental way.

\section{FACTORS AND CONSTRAINTS}

In this section, the factors and constraints that play a role in the DR model are further described. We first have a look at which factors need to be taken into account when reasoning about driver readiness. This is followed by an overview and discussion of measurement technologies that could be used to measure these factors online.

\section{Driver Readiness Factors}

Sheridan and Johannsen [18] give the following model for a human supervisor monitoring the automated vehicle control loop (see Figure 2). The human supervisor monitors the system via displays. Vehicle mode can be changed by control buttons or forced by steering control concepts. The tasks of the human changes much compared to manual driving. Sheridan and Johannsen [18] state that sensomotor activities are less present, whereas vigilance and decision-making behavior becomes more important. For instance, the driver has to plan system actions, set goals and constraints, identify and diagnose system failures and even intervene when necessary [9].

When a driver takes over control and makes the transition from automated driving to the manual loop, his role as supervisor switches back to operator of the vehicle. De Winter et al. [1] identified types of tasks that a driver needs to attend to, and identified which tasks can be attended to at the same time, and which tasks are mutually exclusive. The types of tasks that need to be attended to by the driver when taking back control are:

- Close the longitudinal control loop

- Close the lateral control loop

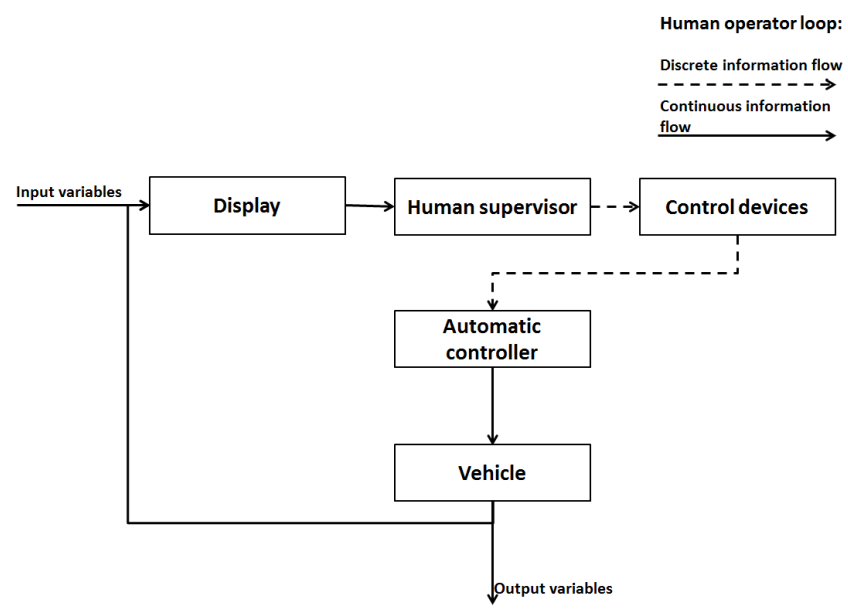

Figure 2. Human supervisor monitoring automated vehicle control loop, adapted from [18].

- Perform a non-visual task (such as for example pressing buttons, steering)

- React to visual stimuli

- Detect and comprehend objects

During automated driving, the driver becomes a supervisor of the system, monitoring whether the system acts according to expectations and intervene if necessary in case of aberrant or emergency situations. The human as a supervisor of automated processes has been a topic of research in many other domains for a long time (e.g., the military domain, aviation, etc.). Reason [17] states that the cognitive structure of humans is not well suited for supervisory control. Important downsides of human supervision of automation are for instance ( $[6,9])$ : Over-reliance, skill degradation, reduced situation awareness, reduced mode awareness, boredom, and attention tunneling. These downsides are serious side effects of automation, leading potentially to disastrous situations if not taken care of in the design and interaction of the automated system or in the training of the human supervisor.

Dufils, Kroon, Hogema, Willemsen, and Sluijsmans [2] looked into several existing projects on automated driving and came up with the following categories of human factor issues:

- Acceptance and comfort depend, among others, on experienced utility, satisfaction, reliability, and trust (e.g., based on the timing of the warning given by automated systems, system settings during transition of control and automated driving). Distrust, discomfort, and lack of acceptance can lead to unwanted driver behavior and traffic safety risks.

- Situation Awareness (SA) concerns the perception, comprehension, and prediction of the elements in the traffic environment by the driver, including system status (cf. [8]). Does the driver understand what the system supports or not, and is he or she aware of his environment when taking over control? Situation awareness is critical when working with autonomous systems for ensuring that they are operating in ways that are consistent with operational goals [3]. 
According to Endsley [4] , situation awareness (SA) is defined as "the perception of the elements in the environment within a volume of time and space, the comprehension of their meaning and the projection of their status in the near future" [4].

- Loss of skill: Loss of driving skills, when one does not need to perform a task for a long time since the automated vehicle took over control, can have an substantial negative effect on safety.

- Behavior adaptation and risk compensation: Drivers may copy automatic driving behavior of vehicle, whilst this behavior might not be safe during manual driving.

- Workload: Driver's workload, both overload and underload, may be an issue with regard to automated driving and safety.

Some recent studies on automated driving focused on takeover times. Zeeb [23] reviewed several papers and found environmental and driver-related factors that influence the takeover time, such as level of distraction, driver expectancy, urgency of the reaction, automation complacency, HMI concept, and age and skill of the driver. It was found that getting physically ready to take-over control was mostly reflexive after an acoustic warning signal. There was no, or marginally, an effect of visual distraction on the time it took to get physically ready. It seemed, however, that the visual and cognitive processing of the situation was affected by driver's state. This review showed that situation awareness is indeed an important factor regarding readiness to take-over control.

For the transition between automated platoon driving to manual driving, reduced SA and mode awareness as a result of boredom, distractions, and performing secondary task (reduced or shifted workload) by the driver was identified as potentially the main hazard [12].

To conclude, after prolonged automated platoon driving it can be expected that the driver is not ready (physically and mentally) to take-over control in an instance. To support the driver in the transition to manual control it is needed to adapt system warnings and feedback to the situation, such that a safe take-over is possible. By adjusting for instance timing of the warning message or the content of the message, SA can be improved, workload is confined, and acceptance and comfort are enhanced. It is therefore needed to assess driver's readiness tot take-over control in relation to the required driver readiness in that situation. Knowing what a driver needs to improve to become ready will make it possible to give the support needed to take-over safely and comfortably.

\section{Monitoring Technologies}

In this section, an overview and discussion of measurement technologies is presented that could be used to measure the factors contributing to driver readiness.

\section{Measuring mental factors contributing to driver readiness}

Above, we have identified several mental factors that contribute to driver readiness. One of them is the SA of the driver. Measuring SA is very challenging. In general, we can differentiate between methods that directly measure SA and methods that try to infer SA from task behavior or performance [7]. Objective measures directly assess SA by gathering data from the individual and comparing this to a ground truth of what is actually happening. An example of this measure is for example SAGAT ('Situation Awareness Global Assessment Technique' [5]). However, these measures can be gathered either in real-time (through 'probes'), during an interruption in task performance, or as post-test after completion of the task, which is not desirable for the problem at hand, as this is very disruptive and cannot be used or accepted as a general means of gathering data during car-driving. The same holds for Subjective measures. Individuals are asked to rate their own SA, which includes an additional task during driving or during the task of taking back control and is very disruptive. Another disadvantage is, that it is not necessarily reliable. Performance measures and behavioral measures infer SA from the task performance and actions the individuals take, respectively, assuming that these good performance and actions are based on a good SA. There are many studies that look into the relationship between driving performance (such as steering movements and vehicle position) and driver state, such as fatigue, distraction, and SA (e.g., [21]). However, when driving automated in a platoon, driving performance measures can not be used to estimate driver state, as the performance and actions of taking over control of the driving task are only measurable after they occurred, it then being too late to support the individual in the task. Measuring psychophysiological aspects assumes a relation between cognitive activity and the physiological state. Examples are the evaluation of an individual's perception of critical environmental cues and the physiological response to upcoming events as a measure of SA. Measures such as heart rate variability, blood pressure, eyelid closure, blinking frequency, and skin conductance may also say something about distraction, stress, attention, workload, and fatigue of the driver [21]. These measures can be measured in-car via sensors (ECG) in steering wheel, wristbands, head and eye trackers, and video recordings without compromising driver comfort [21].

\section{Measuring physical factors contributing to driver readiness}

When a driver drives automatically in a platoon, he or she is free to perform other non-driving related activities, such as doing work on a laptop or sleeping. He does not even have to stay properly seated. It is therefore important that the machine actor assesses whether the driver is physically ready to drive. Pressure sensors in the seat could be used to determine if the driver is seated properly. Sensors in the steering wheel would make it possible to detect whether a driver has both hands on the steering wheel [22]. Also, cameras have been used to determine the driver's hand position on the steering wheel; however, this has been found to not be reliable enough, as the body of the driver may block the view on the steering wheel [22]. Feet positioning is more difficult to measure with pressure sensors since drivers let their feet hover above the gas (and brake) pedal. Cameras would therefore be an outcome to determine whether the feet of the driver are at a ready position and able to take over gas control [22]. 


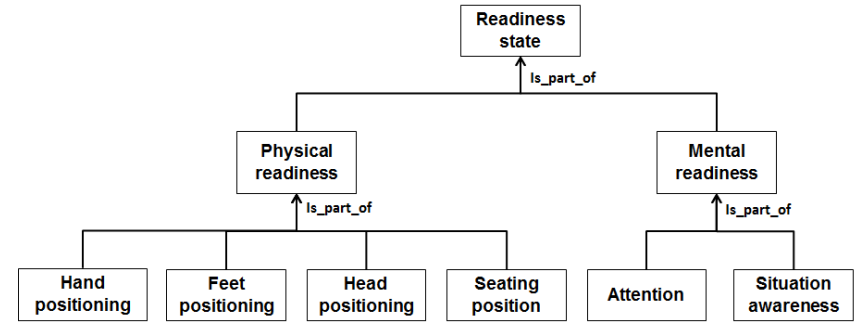

Figure 3. Specification of concepts that play a role for Readiness for the truck driver.

Measuring environmental factors determining required readiness state

Environmental and vehicle measures can be used to estimate the situation on the road in which the driver will have to take over control. The CAN bus of a vehicle may provide information on for instance vehicle speed and the activation of windscreen wipers, which is an indicator for rain. GPS position and input from the navigation device makes it possible to determine current location, type of road and what is expected from the driver when taking over (i.e., taking an exit or merging into a complex motorway junction). Current navigation systems have also an indication of the traffic situation (traffic jams or road works). Future systems as radar and vehicle to roadside/vehicle communication would improve the perception of the traffic and road situation; however, with the current available measures it already is possible to evaluate the environmental factors sufficiently to reason about the readiness of the driver [21].

\section{DRIVER READINESS MODEL}

In this section, the DR model is specified in more detail. In the following, we will first further describe the core factors with their relationships for determining the driver readiness state. This is followed by a specification of the DR model including a reasoning agent to guide the transition of control to the human driver.

\section{Core Factors for Determining the Driver Readiness State}

Several aspects need to be taken into account when determining whether a driver is ready to take over control. As mentioned above, we differentiate between physical readiness and mental readiness, see also Figure 3. Overall, the driver's state of readiness can be seen as a measure of the state of the driver to perform manual driving, which includes being able to attend to the driving tasks as described by De Winter et al. [1], after taking over control.

For the driver to be able to do so, two main aspects need to be defined: Physical and mental readiness. A person is physically ready if the relevant physical aspects regarding the driving task are given. A person is mentally ready if the relevant mental aspects regarding the driving task are given. Both concepts can be further specified, see also Figure 3 .

Physical readiness includes, among others, the following aspects:
- Seating position. The driver should be seated in the driver's seat properly to have free range of motion to reach all vehicle controls.

- Hand positioning. Hands should be positioned such that the driver is able to control the steering wheel and thereby close the lateral control loop.

- Feet positioning. Feet should be positioned such that the driver is able to control the pedals and thereby close the longitudinal control loop.

- Head positioning. Head should be positioned such that the driver is able to react to visual stimuli from inside and outside the car.

Mental readiness includes, among others, the following aspects:

- Attention. Attention is specified in this context to be the selective concentration on a discrete aspect of task execution, while (possibly) ignoring other perceivable information. The absence of attention may be the result of boredom, vigilance or secondary task performance by the driver.

- Situation awareness. For automated driving, SA means that the driver is aware of the traffic situation around him and scanned all relevant aspects of the environment, such that he or she detected all relevant objects and comprehends the situation. A more detailed specification of environmental concepts that play a role in situation awareness regarding automated driving is given in Figure 4).

These concepts combined cover all aspects of the driving task as described by De Winter et al. and therefore provide a complete vision on driver readiness [1].

\section{Overview of CTS Ontology and Agent Behavior}

A general overview of the upper CTS ontology is given in Figure 5. In this ontology, the top level concepts are described, i.e., the stereotypical information that is necessary to reason about general, non-specific, situations. As a consequence, the ontology can be used across domains and applications. For our purpose, the ontology provides generic knowledge about the readiness state to take back control, and about concepts that are necessary to reason about readiness and support the human agent in the transfer of control.

A lower ontology describes the specific instantiation for the driver readiness context of transferring control in truck platooning. In this paper, we will focus on the upper ontology, with presenting examples from the lower ontology of driver readiness in truck platooning.

The goal of CTS ontology is it to be able to reason about the readiness of the driver to take back control, and to advise the driver regarding getting ready to take back control.

For the human-machine system, we differentiate between two different agents (see top right of Figure 5), namely a human actor (in our application domain, this is the driver) and a machine actor (the CTS agent). Both type of agents have situation awareness. The main concept to be reasoned about is the readiness state, which belongs to a human actor, and which 


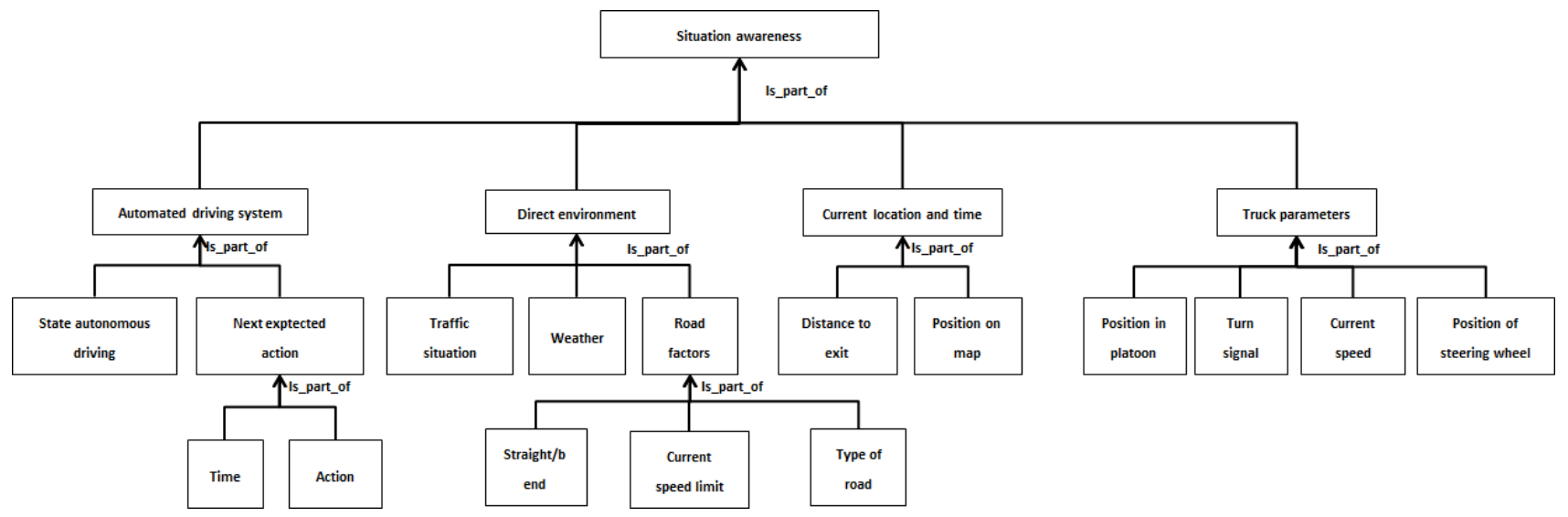

Figure 4. Specification of task-specific concepts that play a role for situation awareness of a truck driver.

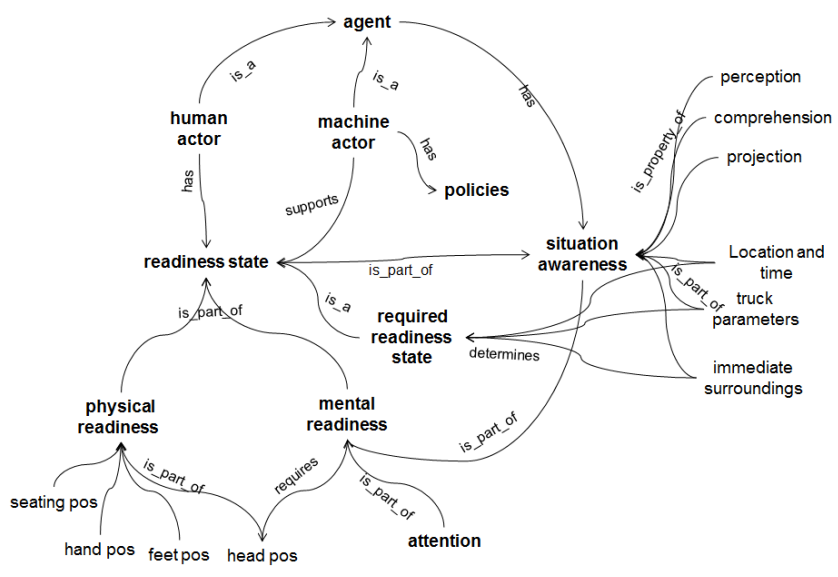

Figure 5. Overview of the CTS ontology.

is supported by the CTS agent. Thi agent supports the transfer of control by setting transfer constraints for the current situation and readiness states (e.g., time), and by enhancing human's readiness when needed (e.g., direction of attention). The readiness state can be seen to represent a measure of readiness, a degree in which specific concepts belonging to readiness are fulfilled.

The Readiness state consists of the two concepts physical readiness and mental readiness, as has been further specified above.

Both the human and the machine actor have situation awareness. Situation awareness is an important concept that is part of the mental readiness of the human actor. Both agents need to have awareness of the driving task, environmental factors such as, for truck platooning the immediate surroundings and the truck parameters, location and time. In addition, for being able to take back control, some self-reflective awareness of one's readiness state to take back control is important for the human driver, as is awareness of the human's readiness state for the machine agent. As described above, situation awareness can be further modeled in three levels (perception, comprehension, and projection [8]). When developing the ontology for driver readiness, the SA-ontology by Smets, Jonker, and Neerincx [19] was partly integrated. The SA-ontology describes the concepts of SA, its effects, how to measure SA and how to use the measurements as support before, during (real-time) and after task execution for evaluation purposes.

The machine CTS agent uses policies to support the transfer of control. Based on the situation awareness of the machine agent, the policies are instantiated to for example adapt the timing of the advice according to the immediate surrounding or the state of the human agent.

The required readiness state is the degree of readiness that the human agent needs to have to safely transfer control from automated system to human agent. The required level is estimated, based on the concerning situational and individal driver factors. The influence these factors have regarding the required readiness state are further specified in the policies. The machine actor supports the human actor based on the difference of the human's readiness state and the required readiness state, based on policies regarding for example presentation of advice.

\section{Policies}

Policies need to be defined that can be used to describe the acceptable boundaries for each concept, per particular scenario, and how the system should act and support the driver in taking back control in this particular scenario [20]. Advantages of using policies are for example re-usability, as policies are domain independent and can apply to many kinds of applications, thus saving development time.

In general, the policies support the machine agent regarding governing issues such as how the system supports the human agent (e.g., presentation of support, timing, and personalization) and how the different concepts are constrained (e.g., the required readiness state is influenced by the particular scenario and task environment), see also Table 1.

An example of a set of policies regarding the timing of support is the following:

1. If the complexity of the task environment is high, the machine agent begins his support of the human agent at an earlier moment before transition of control. 
Table 1. Topics of the machine agent that are governed by policies.

\begin{tabular}{l}
\hline Support of human actor \\
Timing \\
Timing \\
Order \\
Presentation \\
Content \\
Modality \\
Personalization \\
Boundaries of different concepts \\
Boundaries of required readiness state \\
\hline \hline
\end{tabular}

2. If the difference between the current readiness state and the required readiness state is big, the machine agent begins his support at an earlier moment before transition of control.

3. If the human agent's readiness state corresponds to the required readiness state, the machine agent initiates transfer of control to the human agent.

Please note that the policies are specified in a general, domain-independent way. When instantiating the upper ontology in a lower ontology, the policies are instantiated for the application domain.

An instantiation for policy 1, for the truck platooning application, is:

1. If the traffic situation includes high-density traffic, nearby crossroads and/or hindering weather conditions, then begin support of the truck driver early (concerning the time before the actual moment of control transition).

Please note that the degree in which the support will start earlier depends on the traffic situations, and needs to be defined in the lower level ontology (e.g., the metrics for 'high-density traffic', 'nearby crossroads', 'hindering weather conditions', and 'early'). This way, the CTS agent behaviors will be attuned the situation (e.g., rain, snow, fog, backlight) to establish a safe and comfortable transfer.

It should be noted that the highest level of readiness is not always the most preferred level. The optimum is dependent on the momentary driving context and personal characteristics of the driver. The ontology explicates the 'belief-base' and policies, which apply to the context and characteristics and which drive the Control Change Support of the agent. The user interface of the agent should represent these 'drivers' in such a way that the behavior of the CTS-agent is observable, predictable, and directable for the driver [11]. For example, a driver might be able to adapt the required readiness state for a specific trajectory (within specific norms) based on his or her specific experience of a route. This type of flexibility is expected to increase his or her acceptance and to harmonize his or her trust of the CTS-agent.

\section{Truck Platooning Example}

This section will give an example an instantiated driver readiness model for the transfer of control in truck platooning in order to explain the functioning of the CTS model and agent.
A truck driver has been driving in a platoon for 20 minutes on a busy 3 lane motorway and needs to take the next exit. The truck driver is aware he has to take over control in a minute and he has taken a proper seating position, has both hands on the steering wheel, feet near the pedals and head positioned up, being alert. The driver is therefore physically ready to take over control. The eye tracker determines that the driver's level is good by measuring eye blinks and glare. However, the eye tracker also measures that the truck driver did not scan his surroundings and the traffic situation behind him. Since the traffic is busy, the minimum required readiness state for situation awareness includes that the driver has scanned his surroundings in the last 10 seconds before takeover. The machine agent now supports the readiness state of the human agent by addressing the lack of situation awareness of his surroundings, according to his policies, by advising the truck driver to not forget to check his mirrors. The truck driver scans this surroundings and the traffic behind him. The eye tracker measures that the driver scanned his rear and side mirrors; the readiness state of the driver is now in accordance with the required readiness state. The machine agent starts the last phase of giving back control to the driver.

In case the truck driver was sleeping, the machine agent has to support the driver to get physically and mentally ready. First, the truck driver will be warned with a general warning that a take-over is coming up. The truck drive wakes up and gets ready to take over control. However, he forgets to hold the steering wheel with both hands and he does not check his truck parameters. As a consequence, he might not be aware of his current speed and his present location on the map. As a first step, the machine agent gives feedback on the hand positioning. When the driver complies and holds the steering wheel with both hands, the machine agent gives feedback on the truck parameters. The eye tracker determines that the driver took a look at the on-board systems. The required readiness state is now met, after which the machine agent starts the last phase of giving back control to the driver. The policies of the machine agent determine when (order and timing of the support) and how (modality, intensity, message content) the support is given to the truck driver.

However, even with (personalized and adaptive) support, it could be the case that the driver is not ready on time to take over control. In that case, the support agent (by means of the policies) might decide that the take-over does not take place, keeping control with the automation, with possible consequences for example that the vehicle stays in the platoon (and misses the exit), or an emergency stop on the hard shoulder is performed.

\section{Expert Study}

The objective of the ontology is to provide a univocal, coherent and concise knowledge representation for the sensing and influencing of driver readiness, and for guiding the takeover of control (e.g., with sufficien safe-guards). To test the validity and usefulness of the ontology, it was applied to the example described above, and tested with the use of experts (after this validation, we will implement the ontology in the CTS-system, and test the outcomes). Three experts were in- 
terviewed to evaluate the ontology with respect to its clarity, coherence, completeness and consistency: Two were domain and subject-matter experts, and the third an ontology and automation expert. The experts were asked to critically verify the concepts, definitions, and relations of the ontology. In addition, they were asked whether the ontology accommodates the information required for automated advice towards the driver. The comments were, when applicable, directly integrated into the model, and presented to the experts again.

\section{DISCUSSION}

We can identify several challenges regarding both the design of this model and the application for supporting transfer of automation for truck platooning.

\section{Measurements}

As mentioned before, measuring the different factors that play a role for the driver readiness state is a challenge. This holds true for aspects of both physical and mental readiness. Sensing technology, such as eye gaze, heart rate, and facial expressions, is becoming more and more reliable. However, research shows that the technology performance degrades substantially in realistic, dynamic, and complex task environments (in comparison to a lab setup or quiet computer workplace) $[22,13]$. To be able to reason about the readiness of the driver, the measurements need to be reliable enough. An additional challenge for the application in practice is the requirement for real-time sensing and processing of the data. Current tests will provide further insights in the reliability of the present technology (e.g., camera and eye-tracking sensors; see Figure 1).

\section{Configuration}

Another challenge is the configuration of the different concepts, e.g., specifying the required readiness state. Not only does the required readiness state depend on the readiness of the different subfactors (which need to have thresholds regarding an estimation for readiness), but also on the task environment and the individual himself. We will use the concepts specified in the ontology above to use machine learning techniques to learn the different thresholds of being ready. An important aspect of this is also to personalize the values and the policies.

\section{Trust}

To be successful, the CTS system needs to be reliable and the human actor needs to trust the system to deliver relevant and necessary support to increase his readiness. This means that attention needs to be paid to how to treat faulty assessments of the human's readiness of the system. In particular, an evaluation needs to be done regarding preference and handling of false positives (which leads to wrongly assessing the human not to be ready and giving advice on how to get ready) and false negatives (which leads to wrongly assessing the human actor to be ready to take over control). Both influence the human's trust in the system and need to be taken into account in the design of interaction with the human.

\section{Representation of support}

The representation of the support that is given to the human actor is very important, and is specified in the policies. Using policies has the advantage that it is explicitly specified apart from the assessment of driver readiness, and that it can be dynamically adapted regarding the task environment and the user.

Research has to be done on the (multi-) modality of presenting the support to the user, and the timing (order of support, and at which point in time). The particular policies depend on the domain and the task environment, and need to be researched together with domain experts and user studies. For example in truck platooning, support can be given by voice, on a display, or via sounds, haptic information, or lights, directing attention of the driver to particular tasks, perceptions, or actions.

\section{Scope and generalization}

This paper focuses on the development of an ontological Driver Readiness (DR) model and policies for a Control Transfer Support (CTS) agent. The sections above provided a specification of the upper level ontology of the DR model and CTS agent (representing general schemes) and the lower level ontology (representing instantiations of truck platooning with examples). It should be noted that the upper level ontology may not yet cover all important aspects regarding driver readiness and the transfer of control, and that the instantiations do not necessarily cover all possible general behavior patterns as given in the upper level ontology description. We apply a situation cognitive engineering methodology [15] in which the policies and ontology are refined and extended, iteratively. In the next iteration, we will further refine the ontology for completeness.

Furthermore, it should be noted that this paper focuses 'only' on the readiness of the driver in the 'following truck' and that other factors can also have safety effects of truck platoons in traffic. For example, the mutual interactions of platoons with other traffic (including other platoons), and the cognitive and affective load of the driver in the first truck will have an impact on safety. We follow an incremental modular ontology and agent engineering approach, in which the eventual truck platooning system will incorporate a traffic and a task load model, and the corresponding agents to deal with traffic and load factors (e.g., [10]).

\section{CONCLUSIONS}

The ontology presented in this paper provides an univocal, concise and coherent specification of the core concepts of Driver Readiness (DR) and a Control Transfer Support (CTS) agent, along with their relationships. This ontology distinguishes (a) current and required states for the physical (hand, feet, head and seating position) and mental readiness (attention and situation awareness), (b) agents (human and machine actor), (c) policies for agent behaviors, and (d) states of the vehicle and its environment. It provides the knowledge base of the CTS agent that assesses the current and predicted chauffeur state and guides the transition of control in an adaptive and personalized manner. The DR model will be fed by information from the network and in-car sensors. The behaviors of the CTS agent will be generated and constrained by the instantiated policies, providing an important step towards a safe transfer of control from automation to human driver. 
How the ontology supports the development of an automated CTS system was shown in an example. Currently, it is being implemented in an iterative human-centered development process. In addition, the upper level ontology provides some more generic concepts with their relationships that will be applied across domains (e.g., for control hand-overs in a highly automated ship).

In addition to representing the knowledge that is required to develop a CTS system, the ontology also facilitates consistency and clarity of terminology, thus providing a common language among stakeholders, designers, and users. Using an ontology also has the advantage that its format allows non-programmers to add instances and concepts to the ontology, especially when they are working with user-friendly interfaces. The ontology can easily be extended with new instances regarding the domain (e.g., traffic situations, weather aspects), and separates procedural knowledge from declarative knowledge.

The initial version of the ontology was reviewed by experts and subsequently adjusted, and was deemed appropriate for the application investigated in this paper. In future work, the ontology will be extended by the different measurement techniques, and will be validated by further instantiating and implementing it, and evaluating the working of the ontology in a realistic scenario. To be able to do this, we will further refine the different concepts being part of the driver readiness state, and use results of existing simulator studies to define first thresholds for the specification of the required readiness state. We will pay special attention to defining and formalizing the policies to be able to make the system adaptive regarding the task environment and the human actor (we are using the OWL formalism for the specifiation, and Protege as 'tool' to generate and check the ontology).

We plan two type of research activities to increase the applicability and generalization of the current system. In a bottomup approach, we will add new ontological models and agents to our framework based on data-driven trials in the lab and on the road (e.g., for traffic anticipation and for task load harmonization). In a top-down approach, we will further incorporate key elements of relevant theories in the top level ontology (e.g., for situation awareness, cognitive and affective load, attention and trust).

\section{ACKNOWLEDGMENTS}

The research reported in this paper is part of the TNO Early Research Programs (ERP) Human Enhancement (subproject Adaptive Automation) and Applied AI.

\section{REFERENCES}

1. De Winter, J. C., Happee, R., Martens, M. H., and Stanton, N. A. Effects of adaptive cruise control and highly automated driving on workload and situation awareness: A review of the empirical evidence. Transportation research part F: traffic psychology and behaviour 27 (2014), 196-217.

2. Dufils, J. J., Kroon, E. C. M., Hogema, J. H., Willemsen, D. M. C., and Sluijsmans, G. Driving behavior in relation to automation of the driving task. Tech. Rep. TNO 2015 R10157, TNO, Soesterberg, The Netherlands, 2011.

3. Endsley, M. Autonomous horizons: System autonomy in the air force-a path to the future (volume i: Human autonomy teaming). US Department of the Air Force, Washington (2015).

4. Endsley, M. R. Design and evaluation for situation awareness enhancement. In Proceedings of the Human Factors and Ergonomics Society Annual Meeting, vol. 32, SAGE Publications (1988), 97-101.

5. Endsley, M. R. Measurement of situation awareness in dynamic systems. Human Factors: The Journal of the Human Factors and Ergonomics Society 37, 1 (1995), 65-84.

6. Endsley, M. R. Automation and situation awareness. Automation and human performance: Theory and applications (1996), 163-181.

7. Endsley, M. R., and Garland, D. J. Situation awareness analysis and measurement. CRC Press, 2000.

8. Endsley, M. R., and Kiris, E. O. The out-of-the-loop performance problem and level of control in automation. Human Factors: The Journal of the Human Factors and Ergonomics Society 37, 2 (1995), 381-394.

9. Fortmann, F., Suck, S., Cahill, J., Callari, T. C., Javaux, D., and Hasselberg, A. Developing a feedback system to augment monitoring performance of aircraft pilots.

10. Hoedemaeker, M., and Neerincx, M. A. Attuning in-car user interfaces to the momentary cognitive load. In Foundations of Augmented Cognition, 3rd Edition, LNAI 4565 proceedings (2007), 286-293.

11. Johnson, M., Bradshaw, J. M., Feltovich, P. J., Jonker, C. M., Van Riemsdijk, M. B., and Sierhuis, M. Coactive design: Designing support for interdependence in joint activity. Journal of Human-Robot Interaction, 3 (1), 2014 (2014).

12. Martens, M., and van den Beukel, A. The road to automated driving: Dual mode and human factors considerations. In 16th International IEEE Conference on Intelligent Transportation Systems (ITSC 2013), IEEE (2013), 2262-2267.

13. Mioch, T., Giele, T. R. A., Smets, N. J. J. M., and Neerincx, M. A. Measuring emotions of robot operators in urban search and rescue missions. In Proceedings of the 31st European Conference on Cognitive Ergonomics, EACE (2013).

14. Neerincx, M. A., Kaptein, F., van Bekkum, M. A., Krieger, H.-U., Kiefer, B., Peters, R., Broekens, J., Demiris, Y., and Sapelli, M. Ontologies for social, cognitive and affective agent-based support of childs diabetes self-management. In ECAI Workshop on Artificial Intelligence for Diabetes, IOS (2016). 
15. Neerincx, M. A., and Lindenberg, J. Situated cognitive engineering for complex task environments. Ashgate Publishing Limited, 2008.

16. Peeters, M. M., Bosch, K. V. d., Neerincx, M. A., and Meyer, J.-J. C. An ontology for automated scenario-based training. International Journal of Technology Enhanced Learning 6, 3 (2014), 195-211.

17. Reason, J. Human error. Cambridge university press, 1990.

18. Sheridan, T. B. Monitoring behavior and supervisory control, vol. 1. Springer Science \& Business Media, 2013.

19. Smets, N. J. J. M., Jonker, C. M., and Neerincx, M. A. Ontology-based situation awareness support for shared control, extended abstract. In Adjunct Proceedings of 12th Int. Conference on Human-Robot Interaction, ACM/IEEE (2017).

20. Van Diggelen, J., Bradshaw, J. M., Johnson, M., Uszok, A., and Feltovich, P. J. Implementing collective obligations in human-agent teams using kaos policies. In Coordination, Organizations, Institutions and Norms in Agent Systems V. Springer, 2010, 36-52.

21. Wilschut, E., Verbiest, S., von Massow, S., Math, R., Heinrich, A., Ouwerkerk, M., Krishnan, A. B., Terken, J., Tirri, A., Vescovi, M., and de Petris, G. D1 requirements and technology opportunities. state of the art measures for fit to perform. Tech. Rep. D1, EIT Digital Initiative, project Fit 2 Perform, 2016.

22. Wilschut, E., Willemsen, D., Hogema, J., and Martens, M. Adaptive virtual tow bar and transition of control: A truck driving simulator study. Unpublished Manuscript, 2016.

23. Zeeb, K., Buchner, A., and Schrauf, M. What determines the take-over time? an integrated model approach of driver take-over after automated driving. Accident Analysis \& Prevention 78 (2015), 212-221. 